\title{
Process Dimension of Classical and Non-Commutative Processes
}

\author{
Wolfgang Löhr ${ }^{1,2} \quad$ Arleta Szkoła $^{1} \quad$ Nihat Ay ${ }^{1,3}$
}

November 23, 2018

\begin{abstract}
We treat observable operator models (OOM) and their non-commutative generalisation, which we call NC-OOMs. A natural characteristic of a stochastic process in the context of classical OOM theory is the process dimension. We investigate its properties within the more general formulation, which allows to consider process dimension as a measure of complexity of non-commutative processes: We prove lower semi-continuity, and derive an ergodic decomposition formula. Further, we obtain results on the close relationship between the canonical OOM and the concept of causal states which underlies the definition of statistical complexity. In particular, the topological statistical complexity, i.e. the logarithm of the number of causal states, turns out to be an upper bound to the logarithm of process dimension.
\end{abstract}

Keywords: complexity, observable operator models, finitely correlated states, algebraic states, ergodic decomposition, non-commutative processes

\section{Introduction}

The main idea behind various complexity measures, such as statistical complexity, is the same that gave rise to the famous Kolmogorov complexity. Namely, the complexity is the "size" of some minimal "representation" of the object of interest. Different complexity measures are based on different exact definitions of these terms. For Kolmogorov complexity, for instance, representations are Turing machine programs computing individual binary strings, and the size is their length. For statistical complexity ([3]), on the contrary, the objects of interest are probability distributions of stochastic processes instead of individual strings, and the representations are particular kinds of predictive models in the sense of partially deterministic hidden Markov models (HMM). Their size is measured by the Shannon entropy of the internal states of the model.

Observable operator models $(\mathrm{OOM})$ are generative algebraic models that represent a stochastic process. The natural measure of size of an OOM is the dimension of the corresponding real vector space. It is minimal for canonical OOMs of a given stochastic process and was already identified as a characteristic of the process called process dimension. In the present contribution, following the above mentioned reasoning, we want to consider the process dimension as a complexity measure for stochastic processes. We give further indication that this might be appropriate. First, we show the close relation of the canonical OOMs to the concept of causal states which are used to define statistical complexity. Second, we prove that the process dimension, considered as function of the process, is lower semi-continuous. Although there exists no generally accepted axiomatic characterisation of functionals on the space of stochastic processes that quantify complexity, we argue that every complexity measure should feature this property. Indeed, it would be strange to consider a process complex if there is an approximating sequence with (uniformly) simple processes. The natural topology for processes is in this context weak-* topology as opposed to the much stronger variational topology, and lower semi-continuity w.r.t. weak-* topology is a much stronger result.

The construction of causal states relies heavily on conditional probabilities. This makes it difficult to extend the corresponding notion of statistical complexity to the domain of non-commutative processes understood to be states on a quasi-local $\mathrm{C}^{*}$-algebra. The algebraic formulation of OOMs, however, allows to extend the concept of process dimension to the non-commutative setting. Indeed, the construction of finitely correlated states introduced by Fannes et al. in [4] provides OOMs for a class of shift-invariant states on a quasi-local $\mathrm{C}^{*}$-algebra. In the literature, these states are also known as algebraic states. In this paper, however, we refer to their original name. We show lower semi-continuity of the process dimension also in this more general setting.

The outline of our paper is as follows. In Section 2 we present main concepts in the context of classical OOM theory using the terminology introduced by Herbert Jaeger. In particular, process dimension is defined. In Section 3 we review the concept of statistical complexity and the underlying notion of causal states which are defined for classical stationary process. In Proposition 6 we specify the way in which process dimension and causal states are related. As direct implication we obtain an upper bound for, and an ergodic decomposition of the process dimension in the classical case (Corollaries 7 and 8). In Section 4 and 5 we treat non-commutative

\footnotetext{
${ }^{1}$ Max Planck Institute for Mathematics in the Sciences, Inselstraße 22, 04103 Leipzig, Germany

${ }^{2}$ Universität Duisburg-Essen, Universitätsstraße 2, 45141 Essen, Germany

${ }^{3}$ Santa Fe Institute, 1399 Hyde Park Road, Santa Fe, New Mexico 87501, USA
} 
extensions. We start with the definition of NC-OOMs referring to finitely correlated states. As in the classical special case the corresponding process dimension is naturally associated with canonical NC-OOMs. Our main results are contained in Section 5. There we prove lower-semicontinuity and provide an ergodic decomposition formula for the process dimension in the general case.

\section{Classical OOMs (Stochastic Modules)}

Fix a finite set $\Delta$. We consider $\Delta$-valued stochastic processes $X_{\mathbb{N}}:=\left(X_{k}\right)_{k \in \mathbb{N}}$, described by their distributions $P \in \mathcal{P}\left(\Delta^{\mathbb{N}}\right)$, and stationary processes $X_{\mathbb{Z}}$, described by their shift-invariant distributions $P \in \mathcal{P}_{\mathrm{S}}\left(\Delta^{\mathbb{Z}}\right)$.

In [6], Alex Heller introduced a generalisation of functions of Markov chains, called stochastic modules. Later, Herbert Jaeger extended and reformulated this theory in the language of linear algebra ([7]). We use his terminology.

Definition 1. An observable operator model (OOM) with alphabet $\Delta$ is a quadruple $(V, T, v, \ell)$, where $V$ is a real vector space, $T: \Delta \times V \rightarrow V$ is linear in the second argument, $v$ is an element of $V$, and $\ell$ is a linear form on $V$, such that for $T_{d}(v):=T(d, v), n \in \mathbb{N}$ and $d_{1}, \ldots, d_{n} \in \Delta$,

$$
\text { 1. } \ell(v)=1, \quad \text { 2. } \ell \circ \sum_{d \in \Delta} T_{d}=\ell, \quad \text { 3. } P_{d_{1}, \ldots, d_{n}}:=\ell \circ T_{d_{n}} \circ \cdots \circ T_{d_{1}}(v) \geq 0 \text {. }
$$

The vector $v$ is called initial vector, the operators $T_{d}$ are called observable operators and the linear form $\ell$ is called evaluation form ${ }^{1}$. The process $P \in \mathcal{P}\left(\Delta^{\mathbb{N}}\right)$, defined by $P\left(\left[d_{1}, \cdots, d_{n}\right]\right):=P_{d_{1}, \ldots, d_{n}}(n \in \mathbb{N})$, is called generated by the OOM and the dimension $\operatorname{dim}(V)$ of $V$ is called dimension of the OOM.

It is easy to check that the $P_{d_{1}, \ldots, d_{n}}$ are a consistent set of finite-dimensional probabilities. Therefore, by the Kolmogorov extension theorem, the process $P$ well-defined. Every hidden Markov model (HMM) with $n$ internal states canonically induces an $n$-dimensional OOM. For more details, see [7].

Remark. More generally, an HMM with set $\Gamma$ of internal states can be interpreted as OOM with vector space $\mathcal{M}(\Gamma)$ of signed measures of bounded variation on $\Gamma$.

There is a canonical construction of an OOM of a given process $P \in \mathcal{P}\left(\Delta^{\mathbb{N}}\right)$. Let $\mathcal{M}\left(\Delta^{\mathbb{N}}\right)$ be the space of signed measures of bounded variation on $\Delta^{\mathbb{N}}$, i.e. $\mathcal{M}\left(\Delta^{\mathbb{N}}\right)=\operatorname{span}\left(\mathcal{P}\left(\Delta^{\mathbb{N}}\right)\right)$, where span denotes the linear hull. Define the linear maps $\tau_{d}^{\Delta}: \mathcal{M}\left(\Delta^{\mathbb{N}}\right) \rightarrow \mathcal{M}\left(\Delta^{\mathbb{N}}\right)$ by

$$
\tau_{d}^{\Delta}(\mu):=\mu\left([d] \cap \sigma^{-1}(\cdot)\right)
$$

where $\sigma$ is the left-shift on $\Delta^{\mathbb{N}}$. Further define $\ell_{\Delta}: \mathcal{M}\left(\Delta^{\mathbb{N}}\right) \rightarrow \mathbb{R}$ by $\ell_{\Delta}(\mu)=\mu\left(\Delta^{\mathbb{N}}\right)$, i.e. the evaluation form $\ell_{\Delta}$ associates to a measure its total mass. For convenience we define

$$
\tau_{d_{1} \cdots d_{n}}^{\Delta}:=\tau_{d_{n}}^{\Delta} \circ \cdots \circ \tau_{d_{1}}^{\Delta}
$$

Definition 2. For $P \in \mathcal{P}\left(\Delta^{\mathbb{N}}\right)$, let

$$
Q_{P}:=\left\{\tau_{d_{1} \cdots d_{n}}^{\Delta}(P) \mid n \in \mathbb{N}_{0}, d_{1}, \ldots, d_{n} \in \Delta\right\} \quad \text { and } \quad V_{P}:=\operatorname{span}\left(Q_{P}\right) .
$$

For $d \in \Delta$, denote the function $V_{P} \rightarrow V_{P}, \mu \mapsto \tau_{d}^{\Delta}(\mu)$ with a slight abuse of notation again by $\tau_{d}^{\Delta}$. Set $\tau^{\Delta}(d, \mu):=\tau_{d}^{\Delta}(\mu)$. Then $\left(V_{P}, \tau^{\Delta}, P, \ell_{\Delta}\right)$ is called canonical OOM of $P$.

Since $\tau_{d}^{\Delta}\left(V_{P}\right) \subseteq V_{P}$, the canonical OOM is well-defined, and it generates $P$. It has minimal dimension among all OOMs generating $P$, and is, up to isomorphism, unique with this minimality (see [7]). In particular, the dimension of $V_{P}$ is not bigger (but may be essentially smaller) than the minimal number of internal states required for any HMM generating $P$. Another characterisation of $V_{P}$ is in terms of conditional probabilities:

$$
V_{P}=\operatorname{span}\left\{P\left(\sigma^{-n}(\cdot) \mid\left[d_{1}, \ldots, d_{n}\right]\right) \mid n \in \mathbb{N}_{0}, d_{1}, \ldots, d_{n} \in \Delta, P\left(\left[d_{1}, \ldots, d_{n}\right]\right)>0\right\} .
$$

This is true because if we normalise $\tau_{d}^{\Delta}$ pointwise, we obtain the corresponding conditional probability. Note that the normalised version of $\tau_{d}^{\Delta}$ is not linear.

If $A$ is a finite dimensional cylinder set, the same holds for $[d] \cap \sigma^{-1}(A)$. Therefore, $\tau_{d}^{\Delta}$ is weak-* continuous and, consequently, $\tau_{d}^{\Delta}$ maps the weak-* closure ${\overline{V_{P}}}^{w *}$ to itself. Thus $\left(\overline{V_{P}}{ }^{w *}, \tau^{\Delta}, P, \ell_{\Delta}\right)$ is an OOM of $P$, which we call the closed canonical OOM. In the case of finite process dimension, which we are mostly interested in, the canonical OOM and the closed canonical OOM coincide. For considering infinite pasts in the following section, however, the weak-* closure of $V_{P}$ in $\mathcal{M}\left(\Delta^{\mathbb{N}}\right)$ plays a crucial role.

\footnotetext{
${ }^{1}$ Jaeger fixes a basis of $V$ instead of an evaluation form and defines $\ell$ to be the sum of coefficients in the basis expansion.
} 
Example 3 (Canonical OOM and Shift HMM). The (one-sided) shift HMM of $P \in \mathcal{P}\left(\Delta^{\mathbb{N}}\right)$ is a deterministic HMM with set $\Gamma:=\Delta^{\mathbb{N}}$ of internal states. It is in general by no means minimal and it is not possible to restrict it to a smaller subset of $\Gamma$ such that it still generates $P$. If we interpret it as OOM, the internal Vector space is $V=\mathcal{M}\left(\Delta^{\mathbb{N}}\right)$, the associated operators $T_{d}$ are equal to the canonical ones, i.e. $T_{d}=\tau_{d}^{\Delta}$, the initial vector is the initial distribution of the shift HMM, i.e. $v=P$ and the evaluation form is $\mu \mapsto \mu\left(\Delta^{\mathbb{N}}\right)$. Now it is obvious that we can reduce every OOM to a "cyclic" version by restricting $V$ to $\operatorname{span}\left\{T_{d_{1} \ldots d_{n}}(v) \mid\right.$ $\left.n \in \mathbb{N}_{0}, d_{1}, \ldots, d_{n} \in \Delta\right\}$. This reduced shift OOM is just the canonical OOM and thus minimal, but it can in general not be interpreted as HMM.

Definition 4. The process dimension of $P \in \mathcal{P}\left(\Delta^{\mathbb{N}}\right)$ is the dimension of its canonical OOM $\left(V_{P}, \tau^{\Delta}, P, \ell_{\Delta}\right)$ :

$$
\operatorname{dim}(P):=\operatorname{dim}\left(V_{P}\right) \in \mathbb{N} \cup\{\infty\}
$$

The process dimension is derived from a canonical construction and at the same time the minimal dimension necessary for an OOM-representation. Therefore, it is an important internal characteristic of the process and might be considered a complexity measure. As we see in the following section, it is related to statistical complexity.

\section{Infinite Pasts and Causal States}

Now assume we are interested in a stationary process with infinite past, i.e. $X_{\mathbb{Z}}$, described by its shift-invariant distribution $P \in \mathcal{P}_{\mathrm{S}}\left(\Delta^{\mathbb{Z}}\right)$. For simplicity assume that $X_{k}$ is the canonical projection on $\Delta^{\mathbb{Z}}$. We define the canonical OOM of $P$ to be the canonical OOM of its restriction to positive times, i.e. if $P_{\mathbb{N}}:=P \circ X_{\mathbb{N}}^{-1}$ is the distribution of $X_{\mathbb{N}}$ then

$$
\operatorname{dim}(P):=\operatorname{dim}\left(P_{\mathbb{N}}\right)=\operatorname{dim}\left(V_{P_{\mathbb{N}}}\right) .
$$

In [3], the causal states of such a stationary process were introduced and used to define statistical complexity. Causal states are equivalence classes of past trajectories, where two of them are identified if they induce the same conditional probability distribution on the future $X_{\mathbb{N}}$. In this paper we prefer the alternative definition of causal states, where they are measures on the future, i.e. elements of $\mathcal{P}\left(\Delta^{\mathbb{N}}\right)$. This viewpoint was introduced in [8].

If we observe the past $X_{-\mathbb{N}_{0}}$ of $X_{\mathbb{Z}}$, the observation $X_{-\mathbb{N}_{0}}=x_{-\mathbb{N}_{0}}$ induces a certain conditional probability distribution $P\left(X_{\mathbb{N}} \mid X_{-\mathbb{N}_{0}}=x_{-\mathbb{N}_{0}}\right) \in \mathcal{P}\left(\Delta^{\mathbb{N}}\right)$ on the future $X_{\mathbb{N}}$ of the process. The causal state distribution of $P \in \mathcal{P}_{\mathrm{s}}\left(\Delta^{\mathbb{Z}}\right)$ is the distribution of these conditional probabilities. In particular, it is a measure on measures. More precisely we define

Definition 5. Let $P \in \mathcal{P}_{\mathrm{s}}\left(\Delta^{\mathbb{Z}}\right)$. The causal state distribution $\mu_{\mathfrak{C}}^{P} \in \mathcal{P}\left(\mathcal{P}\left(\Delta^{\mathbb{N}}\right)\right)$ of $P$ is defined by

$$
\mu_{\mathfrak{C}}^{P}:=P \circ\left(P\left(X_{\mathbb{N}} \mid X_{-\mathbb{N}_{0}}\right)\right)^{-1}
$$

where we consider $P\left(X_{\mathbb{N}} \mid X_{-\mathbb{N}_{0}}\right)$ to be a (measurable) function from $\Delta^{\mathbb{Z}}$ to $\mathcal{P}\left(\Delta^{\mathbb{N}}\right)$. Further, we define

$$
\mathfrak{C}_{P}:=\operatorname{supp}\left(\mu_{\mathfrak{C}}^{P}\right) \subseteq \mathcal{P}\left(\Delta^{\mathbb{N}}\right)
$$

Remark. a) $\mu_{\mathfrak{C}}^{P}$ is the distribution of the $\mathcal{P}\left(\Delta^{\mathbb{N}}\right)$-valued random variable $P\left(X_{\mathbb{N}} \mid X_{-\mathbb{N}_{0}}\right)$.

b) The causal states correspond to the elements in the image of $P\left(X_{\mathbb{N}} \mid X_{-\mathbb{N}_{0}}\right)$. Thus, the set of causal states depends on the version of conditional probability. $\mathfrak{C}_{P}$, on the other hand, is independent of the choice of conditional probability.

c) The statistical complexity $C_{\mathfrak{C}}(P):=H\left(\mu_{\mathfrak{C}}^{P}\right)$ is the (Shannon) entropy of the causal state distribution. It was originally introduced by Grassberger as true measure complexity in [5].

We obtain the following relation between the causal state distribution and the closed canonical OOM vector space. The weak-* closure of the canonical OOM vector space is equal to the weak-* closure of the vector space spanned by the support $\mathfrak{C}_{P}$ of the causal state distribution. In the finite dimensional case, this means that the two vector spaces are equal. Because the OOM vector space is defined with finite-length pasts and infinite pasts are used for the definition of the causal state distribution, we can interpret this result as follows. Unlike the set of causal states, the canonical OOM vector space is the same if we consider finite or infinite pasts, provided it is finite dimensional. For the infinite dimensional case, the situation is more subtle (see Example 9). Note that $\bar{V}^{w *}$ denotes the closure of $V$ w.r.t. the weak-* topology. Recall that $V_{P}=\operatorname{span}\left(Q_{P}\right)$. 
Proposition 6. Let $P \in \mathcal{P}_{\mathrm{s}}\left(\Delta^{\mathbb{Z}}\right)$. Then

$$
{\overline{V_{P}}}^{w *}=\overline{\operatorname{span}\left(\mathfrak{C}_{P}\right)}{ }^{w *} .
$$

In particular, because finite-dimensional spaces are closed, $\operatorname{dim}(P)=\operatorname{dim}\left(\operatorname{span}\left(\mathfrak{C}_{P}\right)\right)$.

Proof. " $\subseteq$ ": Let $\nu \in Q_{P}$. Then $\nu=\tau_{d_{1} \cdots d_{n}}^{\Delta}\left(P_{\mathbb{N}}\right)$ for some $d_{1}, \ldots, d_{n} \in \Delta$. Define $A$ to be the event that the past is $d_{1}, \ldots, d_{n}$, i.e. $A:=\left\{X_{1-n+k}=d_{k}, k=1, \ldots, n\right\} \subseteq \Delta^{\mathbb{Z}}$. We assume $P(A)>0$, as otherwise $\nu=0$. Further define the non-normalised measure $\widehat{P}:=P(A \cap \cdot) \in \mathcal{M}_{+}\left(\Delta^{\mathbb{Z}}\right)$ and denote $\mu=\widehat{P} \circ\left(P\left(X_{\mathbb{N}} \mid X_{-\mathbb{N}_{0}}\right)\right)^{-1}$. Note that the conditional probability $P\left(X_{\mathbb{N}} \mid X_{-\mathbb{N}_{0}}\right)$ in the definition of $\mu$ is w.r.t. to $P$ not $\widehat{P}$. Using stationarity of $P$ we obtain

$$
\begin{aligned}
\nu & =P_{\mathbb{N}}\left(\left[d_{1}, \ldots, d_{n}\right] \cap \sigma^{-n}(\cdot)\right)=\int P\left(A \cap\left\{X_{\mathbb{N}} \in \cdot\right\} \mid X_{-\mathbb{N}_{0}}\right) \mathrm{d} P=\int_{A} P\left(X_{\mathbb{N}} \mid X_{-\mathbb{N}_{0}}\right) \mathrm{d} P \\
& =\int P\left(X_{\mathbb{N}} \mid X_{-\mathbb{N}_{0}}\right) \mathrm{d} \widehat{P}=\int \operatorname{id}_{\mathcal{P}\left(\Delta^{\mathbb{N}}\right)} \mathrm{d} \mu=\|\mu\| \cdot r\left(\frac{\mu}{\|\mu\|}\right),
\end{aligned}
$$

where id is the identity, $\|\mu\|=\mu\left(\mathcal{P}\left(\Delta^{\mathbb{N}}\right)\right)$ is the norm of total variation and $r: \mathcal{P}\left(\mathcal{P}\left(\Delta^{\mathbb{N}}\right)\right) \rightarrow \mathcal{P}\left(\Delta^{\mathbb{N}}\right)$ is the resultant (also called barycentre map) from integral representation theory. Because $\widehat{P} \ll P$, and thus $\mu \ll \mu_{\mathfrak{C}}^{P}$, the support of $\mu$ is contained in $\mathfrak{C}_{P}$. Due to compactness of $\mathfrak{C}_{P}$, this implies that the barycentre lies in the closed convex hull of $\mathfrak{C}_{P}([2])$, i.e.

$$
r\left(\frac{1}{\|\mu\|} \mu\right) \in \overline{\operatorname{conv}\left(\mathfrak{C}_{P}\right)}{ }^{w *} \quad \text { and } \quad \nu \in{\overline{\operatorname{span}\left(\mathfrak{C}_{P}\right)}}^{w *} .
$$

"?": We have to show that ${\overline{V_{P}}}^{w *}$ has full $\mu_{\mathfrak{C}^{P}}^{P}$-measure, in other words that $P\left(X_{\mathbb{N}} \mid X_{-\mathbb{N}_{0}}\right) \in{\overline{V_{P}}}^{w *} P$-a.s. By the martingale convergence theorem we have for all $B \in \mathfrak{B}\left(\Delta^{\mathbb{N}}\right)$ a.s.:

$$
P\left(\left\{X_{\mathbb{N}} \in B\right\} \mid X_{-\mathbb{N}_{0}}\right)(\omega)=\lim _{n \rightarrow \infty} P\left(\left\{X_{\mathbb{N}} \in B\right\} \mid X_{[-n, 0]}\right)(\omega)=\lim _{n \rightarrow \infty} \frac{\tau_{X_{1}(\omega) \cdots X_{n}(\omega)}^{\Delta}\left(P_{\mathbb{N}}\right)(B)}{P\left(\left[X_{1}(\omega), \ldots, X_{n}(\omega)\right]\right)}
$$

Because $\mathfrak{B}\left(\Delta^{\mathbb{N}}\right)$ is countably generated and setwise (pointwise) convergence of a sequence of probability measures implies weak-* convergence, we obtain $P\left(X_{\mathbb{N}} \mid X_{-\mathbb{N}_{0}}\right) \in \overline{\mathbb{R} \cdot Q_{P}}{ }^{w *} P$-a.s.

Corollary 7. The logarithm of the process dimension is upper bounded by the topological statistical complexity (the logarithm of the number of causal states).

As a second corollary, we obtain an ergodic decomposition formula for process dimension. Namely, the dimension of a process is the sum of the dimensions of its ergodic components. This is not too surprising, because ergodic measures are mutually singular. We prove this formula more generally in the not necessarily commutative case in Section 5. Nevertheless, we give an alternative proof for the classical case here.

Corollary 8. Let $P \in \mathcal{P}_{\mathrm{s}}\left(\Delta^{\mathbb{Z}}\right)$ with ergodic decomposition $\nu \in \mathcal{P}\left(\mathcal{P}_{\mathrm{e}}\left(\Delta^{\mathbb{Z}}\right)\right)$. Then

$$
\operatorname{dim}(P)=\sum_{\mu \in \operatorname{supp}(\nu)} \operatorname{dim}(\mu)
$$

where we use the convention that sums over uncountably many strictly positive elements are infinite.

Proof. We use that $\operatorname{dim}(P)=\operatorname{dim}\left(\operatorname{span}\left(\mathfrak{C}_{P}\right)\right)$ by Proposition 6. It is evident that $\operatorname{dim}(P)$ cannot exceed the sum. Let $P_{1}, \ldots, P_{n} \in \operatorname{supp}(\nu)$ be distinct ergodic components of $P$. Then there are disjoint $A_{1}, \ldots, A_{n} \in$ $\mathfrak{B}\left(\Delta^{\mathbb{N}}\right)$ s.t. $P_{k}\left(\left\{X_{\mathbb{N}} \in A_{k}\right\}\right)=1$. Consequently, $P_{k}\left(\left\{X_{\mathbb{N}} \in A_{k}\right\} \mid X_{-\mathbb{N}_{0}}\right)=1 P_{k}$-a.s. and, because $M_{k}=$ $\left\{\mu \in \mathcal{P}\left(\Delta^{\mathbb{N}}\right) \mid \mu\left(A_{k}\right)=1\right\}$ is closed, $\mathfrak{C}_{P_{k}} \subseteq M_{k}$. The vector spaces $\operatorname{span}\left(M_{k}\right)$ are obviously linearly independent and thus the vector spaces $V_{k}=\operatorname{span}\left(\mathfrak{C}_{P_{k}}\right)$ are linearly independent as well. As $\operatorname{span}\left(\mathfrak{C}_{P}\right) \supseteq$ $\bigcup_{k} V_{k}$, we obtain $\operatorname{dim}(P) \geq \sum_{k} \operatorname{dim}\left(P_{k}\right)$.

Remark. Assume that $\mathfrak{C}_{P}$ is countable and all elements have non-zero $\mu_{\mathfrak{C}}^{P}$-probability, so that we can identify $\mathfrak{C}_{P}$ with the set of causal states. The $\varepsilon$-machine of computational mechanics is an HMM with the set of causal states as internal states. The vector space corresponding to this HMM (if we interpret it as OOM) is $\mathcal{M}\left(\mathfrak{C}_{P}\right)$ as opposed to the canonical OOM vector space $V_{P}=\operatorname{span}\left(\mathfrak{C}_{P}\right)$. The latter can be much lower dimensional, because it utilises the linear structure of $\mathfrak{C}_{P}$. 
The closures in Proposition 6 are really necessary, as we see in the next example. Although $\mathfrak{C}_{P}$ is closed, $\operatorname{span}\left(\mathfrak{C}_{P}\right)$ is not (in general). Also, in general, neither does $\operatorname{span}\left(\mathfrak{C}_{P}\right)$ contain $V_{P}$ nor the other way round.

Example 9. Let $\Delta=\{0,1\}$ and for $p \in[0,1]$ let $P_{p} \in \mathcal{P}_{\mathbf{s}}\left(\Delta^{\mathbb{Z}}\right)$ be the Bernoulli process with parameter $p$, i.e. $P_{p}$ is i.i.d. with $P_{p}\left(\left\{X_{1}=1\right\}\right)=p$. Consider the uncountable mixture $P=\int P_{p} \mathrm{~d} p$, where integration is w.r.t. Lebesgue measure. Then $\mu_{\mathfrak{C}}^{P}$ is the image of Lebesgue measure under the map $p \mapsto P_{p} \circ X_{\mathbb{N}}^{-1}$, and $\mathfrak{C}_{P}=\left\{P_{p} \circ X_{\mathbb{N}}^{-1} \mid p \in[0,1]\right\}$ is the set of i.i.d. processes. We make the following observations:

1. $\operatorname{span}\left(\mathfrak{C}_{P}\right) \cap \mathcal{P}\left(\Delta^{\mathbb{N}}\right)$ is the set of finite mixtures of i.i.d. processes, in particular $\operatorname{span}\left(\mathfrak{C}_{P}\right)$ is not closed.

2. $V_{P}$ has countable algebraic dimension, i.e. it is the linear hull of a countable set, while a basis of $\operatorname{span}\left(\mathfrak{C}_{P}\right)$ has to be uncountable (the family $\left(P_{p}\right)_{p \in[0.1]}$ is linearly independent). Thus, $V_{P}$ cannot contain $\operatorname{span}\left(\mathfrak{C}_{P}\right)$.

3. All elements of $V_{P} \cap \mathcal{P}\left(\Delta^{\mathbb{N}}\right)$ have an uncountable number of ergodic components. Therefore, $\operatorname{span}\left(\mathfrak{C}_{P}\right)$ and $V_{P}$ are even disjoint.

\section{Non-Commutative OOMs}

Since OOMs are, unlike the concept of causal states, formulated algebraically, they have a rather natural generalisation to the setting of non-commutative algebras: Intuitively, we have to replace symbols from an alphabet $\Delta$ by operators representing observables. More precisely, we pass from the algebra $\mathcal{C}(\Delta)$ of (continuous) complex functions on Delta to an operator algebra $\mathcal{A}$ with their self-adjoint operators usually associated with observables of a quantum system. In [4], corresponding models, here referred to as NC-OOMs, have been introduced and investigated in detail for a class of stationary states on quasi-local $\mathrm{C}^{*}$-algebra that feature finite process dimension as introduced in Definition 13 below. In what follows we do not impose this restriction.

Let $\mathcal{A}$ be a finite-dimensional $\mathrm{C}^{*}$-algebra with unit $\mathbb{1}_{\mathcal{A}}$ and positive cone $\mathcal{A}_{+}=\{a \in \mathcal{A} \mid a \geq 0\}$. With $\mathcal{A}_{\mathbb{N}}:=\mathcal{A}^{\otimes \mathbb{N}}$, we denote the $\mathrm{C}^{*}$-algebraic tensor product (i.e. the norm completion of the algebraic tensor product), and similarly, $\mathcal{A}_{I}:=\mathcal{A}^{\otimes I}$ for $I \subseteq \mathbb{Z}$. Let $\mathcal{S}(\mathcal{A})$ denote the set of states on $\mathcal{A}$, i.e. $\mathcal{S}(\mathcal{A})=\{\rho \in$ $\mathcal{A}^{*} \mid \rho$ positive, $\left.\rho\left(\mathbb{1}_{\mathcal{A}}\right)=1\right\}$, where $\mathcal{A}^{*}$ denotes the dual space of $\mathcal{A}$. Note that if $\mathcal{A}=\mathcal{C}(\Delta)=\mathbb{C}^{\Delta}$, then $\mathcal{A}_{\mathbb{N}}=\mathcal{C}\left(\Delta^{\mathbb{N}}\right)$ and $\mathcal{S}\left(\mathcal{A}_{\mathbb{N}}\right)$ can be identified with $\mathcal{P}\left(\Delta^{\mathbb{N}}\right)$.

Definition 10. $(V, T, v, \ell)$ is an NC-OOM with output algebra $\mathcal{A}$ if $V$ is a vector space, $T: \mathcal{A} \times V \rightarrow V$, $(a, w) \mapsto T_{a}(w)$ is bilinear, $v \in V$ and $\ell \in V^{*}$ such that for $n \in \mathbb{N}, a_{1}, \ldots, a_{n} \in \mathcal{A}_{+}$

$$
\text { 1. } \ell(v)=1, \quad \text { 2. } \ell \circ T_{\mathbb{1}_{\mathcal{A}}}=\ell, \quad 3 . \ell \circ T_{a_{n}} \circ \cdots \circ T_{a_{1}}(v) \geq 0 .
$$

The state $\varphi$ on $\mathcal{A}_{\mathbb{N}}$ obtained by linear extension of $\varphi\left(a_{1} \otimes \cdots \otimes a_{n}\right):=\ell \circ T_{a_{n}} \circ \cdots \circ T_{a_{1}}(v)$ is called generated by the NC-OOM.

Remark. a) The state $\varphi$ generated by an NC-OOM is a well defined state on $\mathcal{A}_{\mathbb{N}}$. Note that it is not necessarily translation invariant.

b) We adapted the definition of finitely correlated states given in [4], to fit the classical OOM definition, see Definition 1. In [4], the $T_{a_{k}}$ are applied in reverse order and $\ell$ need not be normalised: there $\varphi\left(a_{1} \otimes \cdots \otimes\right.$ $\left.a_{n}\right)=\frac{1}{\ell(e)} \cdot \ell \circ T_{a_{1}} \circ \cdots \circ T_{a_{n}}(v)$. Note that as a consequence of the reverse order combined with condition 2. the associated finitely correlated states in [4] are translation invariant by construction:

$$
\begin{aligned}
\varphi\left(\mathbb{1}_{\mathcal{A}} \otimes a_{1} \otimes \cdots \otimes a_{n}\right) & =\frac{1}{\ell(v)} \ell \circ T_{\mathbb{1}_{\mathcal{A}}} \circ T_{a_{1}} \circ \cdots \circ T_{a_{n}}(v) \\
& =\frac{1}{\ell(v)} \ell \circ T_{a_{1}} \circ \cdots \circ T_{a_{n}}(v) \\
& =\varphi\left(a_{1} \otimes \cdots \otimes a_{n}\right)
\end{aligned}
$$

Let $\mathcal{M}_{\mathbb{C}}\left(\Delta^{\mathbb{N}}\right)$ be the set of complex-valued measures of bounded variation on $\Delta^{\mathbb{N}}$, and $\psi: \mathcal{M}_{\mathbb{C}}\left(\Delta^{\mathbb{N}}\right) \rightarrow$ $\mathcal{C}\left(\Delta^{\mathbb{N}}\right)^{*}$ the natural isomorphism. Then every probability measure $P \in \mathcal{P}\left(\Delta^{\mathbb{N}}\right)$ corresponds to the state $\psi(P)$ on the commutative $C^{*}$-algebra $\mathcal{C}\left(\Delta^{\mathbb{N}}\right)$. In the same vein, OOMs with output alphabet $\Delta$ can be interpreted as the special case of NC-OOMs with commutative output algebra $\mathcal{A}=\mathcal{C}(\Delta)$. More precisely, there is a natural one-to-one correspondence $\iota$ as follows. If $\mathcal{O}=\left(V,\left(T_{d}\right)_{d \in \Delta}, v, \ell\right)$ is an OOM, the corresponding NC-OOM is $\iota(\mathcal{O})=(\widetilde{V}, T, v, \tilde{\ell})$, where $\widetilde{V}=V \oplus i V$ is the complexification of $V$, and $\tilde{\ell}$ is the complex-linear extension of $\ell$ to $\widetilde{V}$. $T$ is given by $T(f, w):=\sum_{d \in \Delta} f(d) T_{d}(w)$ for $f \in \mathcal{A}=\mathcal{C}(\Delta), w \in V$, and extended linearly to 
$w \in \widetilde{V}$. Obviously, $\operatorname{dim}_{\mathbb{R}}(V)=\operatorname{dim}_{\mathbb{C}}(\widetilde{V})$. Furthermore, it is straight-forward to check that if $\mathcal{O}$ generates $P \in \mathcal{P}\left(\Delta^{\mathbb{N}}\right)$, denoted by gen $(\mathcal{O})=P$, then $\iota(\mathcal{O})$ generates $\psi(P) \in \mathcal{S}\left(\mathcal{A}_{\mathbb{N}}\right)$, denoted by gen $(\iota(\mathcal{O}))=\psi(P)$. This means that the following diagram commutes:

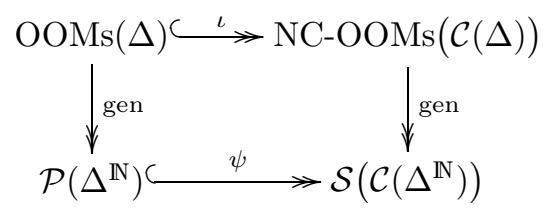

The canonical NC-OOM of a state $\varphi \in \mathcal{S}\left(\mathcal{A}_{\mathbb{N}}\right)$ is defined similarly to the canonical OOM of a classical probability distribution, cf. Definition 2. In more detail, the dual $\mathcal{A}_{\mathbb{N}}^{*}$ of $\mathcal{A}_{\mathbb{N}}$ corresponds to the $\operatorname{space} \mathcal{M}\left(\Delta^{\mathbb{N}}\right)$ of signed measures used in the classical construction. The initial vector is $\varphi$ itself, and the evaluation functional $\ell_{\mathcal{A}}$ is the evaluation at $\mathbb{1}_{\mathcal{A}_{\mathbb{N}}}$, i.e. $\ell_{\mathcal{A}}(\rho)=\rho\left(\mathbb{1}_{\mathcal{A}_{\mathbb{N}}}\right)$. The map $\tau^{\mathcal{A}}: \mathcal{A} \times \mathcal{A}_{\mathbb{N}}^{*} \rightarrow \mathcal{A}_{\mathbb{N}}^{*}$ is defined by

$$
\tau^{\mathcal{A}}(a, \rho):=\tau_{a}^{\mathcal{A}}(\rho):=\rho(a \otimes \cdot):=(X \mapsto \rho(a \otimes X))
$$

and again we set

$$
\tau_{a_{1} \cdots a_{n}}^{\mathcal{A}}:=\tau_{a_{n}}^{\mathcal{A}} \circ \cdots \circ \tau_{a_{1}}^{\mathcal{A}}
$$

Definition 11. For $\varphi \in \mathcal{S}\left(\mathcal{A}_{\mathbb{N}}\right)$ let

$$
V_{\varphi}:=\operatorname{span}\left\{\tau_{a_{1} \cdots a_{n}}^{\mathcal{A}}(\varphi) \mid n \in \mathbb{N}_{0}, a_{1}, \ldots, a_{n} \in \mathcal{A}\right\} \subseteq \mathcal{A}_{\mathbb{N}}^{*}
$$

and denote the function $\mathcal{A} \times V_{\varphi} \rightarrow V_{\varphi},(a, \rho) \mapsto \tau_{a}^{\mathcal{A}}(\rho)$ with a slight abuse of notation again by $\tau^{\mathcal{A}}$. Then $\left(V_{\varphi}, \tau^{\mathcal{A}}, \varphi, \ell_{\mathcal{A}}\right)$ is called canonical NC-OOM of $\varphi$.

Remark. a) $V_{\varphi}$ is a vector space and $\tau_{a}^{\mathcal{A}}$, for all $a \in \mathcal{A}$, maps $V_{\varphi}$ into $V_{\varphi}$.

b) The discussion in [4] is about translation invariant states on $\mathcal{A}_{\mathbb{Z}}$. There, the image $W_{\varphi}$ of the map $\mathcal{A}_{-\mathbb{N}_{0}} \rightarrow \mathcal{A}_{\mathbb{N}}^{*}, a \mapsto \varphi(a \otimes \cdot)$ is used instead of $V_{\varphi}$. In general, we have the relation $V_{\varphi} \subseteq W_{\varphi} \subseteq{\overline{V_{\varphi}}}^{w *}$. In the finite-dimensional case, however, the two spaces coincide.

c) Let $P \in \mathcal{P}\left(\Delta^{\mathbb{N}}\right)$, and $\mathcal{O}$ the canonical OOM of $P$. Then the corresponding NC-OOM $\iota(\mathcal{O})$ is the canonical NC-OOM of $\psi(P)$ up to the identification of $\mathcal{M}_{\mathbb{C}}\left(\Delta^{\mathbb{N}}\right)$ with $\mathcal{C}\left(\Delta^{\mathbb{N}}\right)^{*}$ by the isomorphism $\psi$. In particular, $\tau_{a}^{\mathcal{A}}=\sum_{d \in \Delta} a(d) \cdot \psi \circ \tau_{d}^{\Delta} \circ \psi^{-1}$ and

$$
\operatorname{dim}(P)=\operatorname{dim}(\psi(P))
$$

d) Note that $\tau_{a}^{\mathcal{A}}$ is weak-* continuous.

Lemma 12. The canonical $N C$-OOM of $\varphi \in \mathcal{S}\left(\mathcal{A}_{\mathbb{N}}\right)$ generates $\varphi$.

Proof. Let $a_{1}, \ldots, a_{n} \in \mathcal{A}$. We obtain

$$
\ell_{\mathcal{A}} \circ \tau_{a_{1} \ldots a_{n}}^{\mathcal{A}}(\varphi)=\tau_{a_{n}}^{\mathcal{A}}\left(\tau_{a_{1} \ldots a_{n-1}}^{\mathcal{A}}(\varphi)\right)\left(\mathbb{1}_{\mathcal{A}_{\mathbb{N}}}\right)=\tau_{a_{1} \ldots a_{n-1}}^{\mathcal{A}}(\varphi)\left(a_{n}\right)=\cdots=\varphi\left(a_{1} \otimes \cdots \otimes a_{n}\right) .
$$

Similarly to the definition of process dimension of a probability distribution as given in Definition 4 , we propose:

Definition 13. The process dimension of $\varphi \in \mathcal{S}\left(\mathcal{A}_{\mathbb{N}}\right)$ is the dimension of its canonical NC-OOM:

$$
\operatorname{dim}(\varphi):=\operatorname{dim}\left(V_{\varphi}\right) \in \mathbb{N} \cup\{\infty\}
$$

\section{Properties of Process Dimension}

In this section we present our main results: lower semi-continuity and an ergodic decomposition formula for process dimension. In the classical special case, corresponding results for a class of complexity measures have been obtained in $[9,8]$. For the technical prerequisits that are required for our non-commutative extension we refer to the books $[1,10]$.

Theorem 14. The process dimension $\operatorname{dim}: \mathcal{S}\left(\mathcal{A}_{\mathbb{N}}\right) \rightarrow \mathbb{N} \cup\{\infty\}$ is weak-* lower semi-continuous. 
Proof. Because $\mathcal{A}_{\mathbb{N}}$ is separable, $\mathcal{S}\left(\mathcal{A}_{\mathbb{N}}\right)$ is weak-* metrisable and thus sequential semi-continuity implies semi-continuity. Let $\varphi$ be the weak-* limit of a sequence $\left(\varphi_{n}\right)_{n \in \mathbb{N}}$ in $\mathcal{S}\left(\mathcal{A}_{\mathbb{N}}\right)$ and $\operatorname{dim}(\varphi) \geq d$. We have to show that $\operatorname{dim}\left(\varphi_{n}\right) \geq d$ for sufficiently large $n$. Let $\left(V_{\varphi}, \tau^{\mathcal{A}}, \varphi, \ell_{\mathcal{A}}\right)$ be the canonical NC-OOM of $\varphi$. Since, by Definition $11, \operatorname{dim}\left(V_{\varphi}\right)=\operatorname{dim}(\varphi)$, we can choose linearly independent $v_{1}, \ldots, v_{d} \in V_{\varphi}$. Moreover, by definition of $V_{\varphi}$, there exist $a_{k j} \in \mathcal{A}, 1 \leq k \leq d, 1 \leq j \leq m_{k}$, such that $v_{k}=\tau_{A_{k 1} \ldots A_{k m_{k}}}^{\mathcal{A}}(\varphi)$. For $n \in \mathbb{N}$, we define vectors $v_{k}^{n}:=\tau_{A_{k 1} \ldots A_{k m_{k}}}^{\mathcal{A}}\left(\varphi_{n}\right)$ in $V_{\varphi_{n}}$, respectively. Due to continuity of $\tau_{A}^{\mathcal{A}}$, we have $v_{k}^{n} \stackrel{*}{\rightarrow} v_{k}$. If $v_{1}^{n}, \ldots, v_{d}^{n}$ are linearly independent for all sufficiently large $n$, the proof is finished. Suppose this is not the case and w.l.o.g. that they are dependent for all $n$. Then there are $\lambda_{k}^{n} \in[-1,1]$ with $\max _{k}\left|\lambda_{k}^{n}\right|=1$ and $\sum_{k=1}^{d} \lambda_{k}^{n} v_{k}^{n}=0$ for all $n$. Because $[-1,1]^{d}$ is compact, we may assume by passing to a subsequence that $\lambda_{k}^{n} \stackrel{n \rightarrow \infty}{\longrightarrow} \lambda_{k}$ for some $\lambda_{k}$. Due to weak-* continuity of addition and scalar multiplication, $\sum_{k} \lambda_{k} v_{k}=0$ and hence $\lambda_{k}=0$ for all $k$, in contradiction to $\max _{k}\left|\lambda_{k}^{n}\right|=1$.

Due to the one-to-one correspondence between canonical OOMs of classical processes and canonical NCOOMs of associated states on (abelian) $\mathrm{C}^{*}$-algebras the above theorem has the following corollary.

Corollary 15. The classical process dimension $\operatorname{dim}: \mathcal{P}\left(\Delta^{\mathbb{N}}\right) \rightarrow \mathbb{N} \cup\{\infty\}$ is weak-* lower semi-continuous.

We now derive an ergodic decomposition formula for process dimension in the stationary case. Let $\mathcal{S}_{\mathrm{s}}\left(\mathcal{A}_{\mathbb{N}}\right)$ be the convex set of translation invariant states, and $\mathcal{S}_{\mathrm{e}}\left(\mathcal{A}_{\mathbb{N}}\right) \subseteq \mathcal{S}_{\mathrm{s}}\left(\mathcal{A}_{\mathbb{N}}\right)$ the set of ergodic states, i.e. extreme points in $\mathcal{S}_{\mathrm{S}}\left(\mathcal{A}_{\mathbb{N}}\right)$. Since $\mathcal{A}_{\mathbb{N}}$ is asymptotically abelian (w.r.t. the shift) and $\mathcal{S}_{\mathrm{S}}\left(\mathcal{A}_{\mathbb{N}}\right)$ is metrisable, $\mathcal{S}_{\mathrm{s}}\left(\mathcal{A}_{\mathbb{N}}\right)$ is a simplex and the set $\mathcal{S}_{\mathrm{e}}\left(\mathcal{A}_{\mathbb{N}}\right)$ of ergodic states is measurable in $\mathcal{S}_{\mathrm{S}}\left(\mathcal{A}_{\mathbb{N}}\right)$. In particular, every translation invariant state $\varphi$ has a unique ergodic decomposition $\nu \in \mathcal{P}\left(\mathcal{S}_{\mathrm{s}}\left(\mathcal{A}_{\mathbb{N}}\right)\right)$, which is supported by the ergodic states, $\nu\left(\mathcal{S}_{\mathrm{e}}\left(\mathcal{A}_{\mathbb{N}}\right)\right)=1$, and $\varphi$ is the barycentre of $\nu$,

$$
\varphi=\int_{\mathcal{S}_{\mathrm{e}}\left(\mathcal{A}_{\mathbb{N}}\right)} \operatorname{id~} \mathrm{d} \nu .
$$

Moreover, in what follows, we make use of the important fact that in our situation the ergodic decomposition is orthogonal. For details, see [1, Sec. 4.1, 4.3.1]. We obtain the following ergodic decomposition formula for process dimension.

Theorem 16. Let $\varphi \in \mathcal{S}_{\mathrm{s}}\left(\mathcal{A}_{\mathbb{N}}\right)$ be a translation invariant state with ergodic decomposition $\nu \in \mathcal{P}\left(\mathcal{S}_{\mathrm{e}}\left(\mathcal{A}_{\mathbb{N}}\right)\right)$. Then

$$
\operatorname{dim}(\varphi)=\sum_{\psi \in \operatorname{supp}(\nu)} \operatorname{dim}(\psi)
$$

For the proof, we use the following two lemmas.

Lemma 17 (representation on Hilbert space). Let $\varphi \in \mathcal{S}_{\mathrm{s}}\left(\mathcal{A}_{\mathbb{N}}\right)$ and $\left(\mathcal{H}_{\varphi}, \pi_{\varphi}, \xi_{\varphi}\right)$ be the GNS-representation of $\mathcal{A}_{\mathbb{N}}$ w.r.t. $\varphi$. Then there is a linear injection $\iota_{\varphi}$ from $V_{\varphi}$ into $\mathcal{H}_{\varphi}$ with

$$
\rho(X)=\left\langle\pi_{\varphi}(X) \iota_{\varphi}(\rho), \xi_{\varphi}\right\rangle_{\varphi} \quad \forall X \in \mathcal{A}_{\mathbb{N}}, \rho \in V_{\varphi}
$$

Proof. Let $u$ be the unitary representation of the shift on $\mathcal{A}_{\mathbb{N}}$. Then stationarity of $\varphi$ implies $u \xi_{\varphi}=\xi_{\varphi}$. For $a_{1}, \ldots, a_{n} \in \mathcal{A}$ and $A=a_{1} \otimes \cdots \otimes a_{n}$ let

$$
\xi_{A}:=u \pi_{\varphi}\left(a_{n}\right) \cdots u \pi_{\varphi}\left(a_{1}\right) \xi_{\varphi}=u^{n} \pi_{\varphi}(A) \xi_{\varphi} \in \mathcal{H}_{\varphi}
$$

and extend the definition to $A \in \mathcal{A}_{\{1, \ldots, n\}}$ linearly. Then we have for $A=\sum_{i=1}^{m} a_{i 1} \otimes \cdots \otimes a_{i n}$

$$
\tau_{A}^{\mathcal{A}}(\varphi)(X):=\sum \tau_{a_{i 1} \cdots a_{i n}}^{\mathcal{A}}(\varphi)(X)=\left\langle\left(u^{n}\right)^{*} \pi_{\varphi}(X) u^{n} \pi_{\varphi}(A) \xi_{\varphi}, \xi_{\varphi}\right\rangle_{\varphi} \stackrel{\left(u \xi_{\varphi}=\xi_{\varphi}\right)}{=}\left\langle\pi_{\varphi}(X) \xi_{A}, \xi_{\varphi}\right\rangle_{\varphi}
$$

where $\tau_{a_{i 1} \cdots a_{i n}}^{\mathcal{A}}$ is defined in (1). For every $\rho \in V_{\varphi}$, there is an $n \in \mathbb{N}$ and $A \in \mathcal{A}_{<\infty}:=\bigcup_{n \in \mathbb{N}} \mathcal{A}_{\{1, \ldots, n\}}$ with $\rho=\tau_{A}^{\mathcal{A}}(\varphi)$. Define $\iota_{\varphi}(\rho):=\xi_{A}$. Because $\xi_{\varphi}$ is cyclic and $\mathcal{A}_{<\infty}$ is dense in $\mathcal{A}_{\mathbb{N}},\left\langle\pi_{\varphi}(X) \zeta_{1}, \xi_{\varphi}\right\rangle_{\varphi}=$ $\left\langle\pi_{\varphi}(X) \zeta_{2}, \xi_{\varphi}\right\rangle_{\varphi}$ for all $X \in \mathcal{A}_{<\infty}$ implies that $\zeta_{1}=\zeta_{2}$. Thus $\iota_{\varphi}$ is well-defined. Injectivity is obvious, because $\rho$ can be recovered from $\xi_{A}$ by (2).

Lemma 18. Let $\varphi \in \mathcal{S}_{\mathrm{S}}\left(\mathcal{A}_{\mathbb{N}}\right)$ and $\varphi=\sum_{\psi \in \Psi} \nu(\psi) \psi$, where $\Psi \subset \mathcal{S}_{\mathrm{s}}\left(\mathcal{A}_{\mathbb{N}}\right)$ is countable and $\nu(\psi)>0$. Then

$$
{\overline{V_{\varphi}}}^{w *}={\overline{\sum_{\psi \in \Psi} V_{\psi}}}^{w *}
$$

where $V_{\varphi}$ is defined in Definition 11. 
Proof. "ᄃ": By linearity of $\tau_{a}^{\mathcal{A}}$, we obviously have $V_{\varphi} \subseteq \sum_{\psi} V_{\psi}$.

" $\supseteq$ ": Because $\nu(\psi) \psi \leq \varphi$, there is a $\xi_{\psi} \in \mathcal{H}_{\varphi}$ with $u \xi_{\psi}=\xi_{\psi}$ and $\psi(X)=\left\langle\pi_{\varphi}(X) \xi_{\psi}, \xi_{\varphi}\right\rangle_{\varphi}$. As $\xi_{\varphi}$ is cyclic and $\mathcal{A}_{<\infty}$ is dense in $\mathcal{A}_{\mathbb{N}}$, there is a sequence $A_{n} \in \mathcal{A}_{\{1, \ldots, n\}}$ with $\pi_{\varphi}\left(A_{n}\right) \xi_{\varphi} \rightarrow \xi_{\psi}$. Let $\rho_{n}:=\tau_{A_{n}}^{\mathcal{A}}(\varphi)$. Then $\rho_{n} \in V_{\varphi}$, and for all $X \in \mathcal{A}_{\mathbb{N}}$

$$
\rho_{n}(X)=\langle\underbrace{\pi_{\varphi}(X) u^{n}}_{\|\cdot\|=\left\|\pi_{\varphi}(X)\right\|<\infty} \pi_{\varphi}\left(A_{n}\right) \xi_{\varphi}, \xi_{\varphi}\rangle_{\varphi} \stackrel{n \rightarrow \infty}{\longrightarrow}\left\langle\pi_{\varphi}(X) \xi_{\psi}, \xi_{\varphi}\right\rangle_{\varphi}=\psi(X) .
$$

Thus $\rho_{n} \stackrel{*}{\rightarrow} \psi$ and $\psi \in{\overline{V_{\varphi}}}^{w *}$. As $\tau_{a}^{\mathcal{A}}$ is weak-* continuous, $\tau_{a}^{\mathcal{A}}\left({\overline{V_{\varphi}}}^{w *}\right) \subseteq{\overline{V_{\varphi}}}^{w *}$, hence $V_{\psi} \subseteq{\overline{V_{\varphi}}}^{w *}$.

The lemma shows that in order to represent $\varphi$ in terms of NC-OOMs, we have to represent all ergodic components $\psi$ of $\varphi$ (and not more). We still have to show that they have to be represented independently without synergies. This follows easily from the orthogonality of the ergodic decomposition.

Proof of Theorem 16. 1. Finitely many ergodic components: In this case, Lemma 18 directly implies " $\leq . "$ For " $\geq$," we may assume that $\operatorname{dim}\left(V_{\varphi}\right)<\infty$ and thus also $\operatorname{dim}\left(V_{\psi}\right)<\infty$ for all ergodic components $\psi$. In particular, $V_{\varphi}=\sum_{\psi} V_{\psi}$ by Lemma 18. We can identify the GNS-Hilbert space $\mathcal{H}_{\psi}$ with a subspace of $\mathcal{H}_{\varphi}$ and because the ergodic decomposition is orthogonal, the $\mathcal{H}_{\psi}$ are mutually orthogonal. Since $\iota_{\varphi}\left(V_{\psi}\right) \subseteq \mathcal{H}_{\psi}$, by Lemma 17 , the sum of vector spaces is direct, i.e. $V_{\varphi}=\bigoplus_{\psi} V_{\psi}$, and $\operatorname{dim}\left(V_{\varphi}\right)=$ $\sum_{\psi} \operatorname{dim}\left(V_{\psi}\right)$.

2. Infinitely many ergodic components: The sum on the right-hand side is infinite. To see that also $\operatorname{dim}(\varphi)=\infty$, fix $n \in \mathbb{N}$ and choose a decomposition of $\operatorname{supp}(\nu)$ into disjoint measurable subsets $\Psi_{k}$, $k=1, \ldots, n$ with positive $\nu$-measure. Define $\psi_{k}:=\int_{\Psi_{k}}$ id d $\nu$ to be the barycentre of $\nu \uparrow_{\Psi_{k}}$. Then the decomposition $\psi=\sum_{k} \psi_{k}$ is orthogonal and by the above $\operatorname{argument} \operatorname{dim}\left(V_{\varphi}\right)=\sum_{k} \operatorname{dim}\left(V_{\psi_{k}}\right) \geq n$.

Note that Theorem 16 provides an alternative proof of Corollary 8.

\section{Acknowledgement}

This work has been supported by the Santa Fe Institute.

\section{References}

[1] Ola Bratteli and Derik W. Robinson. Operator Algebras and Quantum Statistical Mechanics 1. Springer, second edition, 1987.

[2] Gustave Choquet. Lectures on Analysis, Volume II (Representation Theory). W. A. Benjamin, Inc., 1969.

[3] James P. Crutchfield and Karl Young. Inferring statistical complexity. Phys. Rev. Let., 63:105-108, 1989.

[4] M. Fannes, B. Nachtergaele, and R. F. Werner. Finitely correlated states on quantum spin chains. Commun. Math. Phys., 144(3):443-490, 1992.

[5] Peter Grassberger. Toward a quantitative theory of self-generated complexity. Int. J. Theor. Phys., 25:907-938, 1986.

[6] Alex Heller. On stochastic processes derived from Markov chains. Annals of Mathematical Statistics, $36: 1286-1291,1965$.

[7] Herbert Jaeger. Observable operator models for discrete stochastic time series. Neural Computation, 12(6):1371-1398, 2000.

[8] Wolfgang Löhr. Properties of the statistical complexity functional and partially deterministic HMMs. Entropy, 11(3):385-401, 2009.

[9] Wolfgang Löhr. Models of Discrete-Time Stochastic Processes and Associated Complexity Measures. PhD thesis, University of Leipzig, 2010. http://nbn-resolving.de/urn:nbn:de:bsz:15-qucosa-38267.

[10] David Ruelle. Statistical Mechanics, rigorous results. World Scientific, 1969. 